\title{
PREVALÊNCIA DE DOR MUSCULOESQUELÉTICA EM \\ CORREDORES DE RUA NO MOMENTO EM QUE PRECEDE O INÍCIO DA CORRIDA
}

\author{
GRAD. TIÊ PARMA YAMATO \\ Mestranda do Programa de Mestrado em Fisioterapia da \\ Universidade Cidade de São Paulo (São Paulo - São Paulo - Brasil) \\ Membro do Grupo de Estudos em Lesões da Corrida SPRunlG (São Paulo Running Injury Group) \\ e-mail: ti_parma@hotmail.com
}

GRAD. BRUNO TIROTTI SARAGIOTTO

Mestrando do Programa de Mestrado em Fisioterapia da Universidade Cidade de São Paulo (São Paulo - São Paulo - Brasil)

Membro do Grupo de Estudos em Lesões da Corrida SPRunIG (São Paulo Running Injury Group) e-mail: bruno.saragiotto@gmail.com

\section{DR. ALEXANDRE DIAS LOPES}

Doutor em Biodinâmica do Movimento Humano

Professor do programa de mestrado da UNICID (São Paulo - São Paulo - Brasil)

Membro do Grupo de Estudos em Lesões da Corrida SPRunIG (São Paulo Running Injury Group) e-mail: aledlopes@yahoo.com.br

\section{RESUMO}

A participação em corridas de rua vem aumentando significativamente nos últimos anos, motivada pelos beneficios a saúde, fácil execução e baixo custo. Os objetivos desse estudo foram: analisar a prevalência de dor musculoesquelética em corredores de rua no momento em que precede o início da prova, além de discutir alguns fatores etiológicos relacionados à prática da corrida. Os participantes foram divididos em dois grupos, um grupo foi composto por corredores com queixa de dor musculoesquelética e outro sem queixa. Foi constatada uma diferença estatisticamente significante quando comparados o número de treinos por semana $(p=0,03)$ e a quilometragem semanal percorrida $(p=0,01)$. Concluímos que a prevalência de dor foi de aproximadamente $25 \%$ e estava diretamente associada ao volume de treino semanal.

PALAVRAS-CHAVE: Corrida; dor; prevalência; esporte. 


\section{INTRODUÇÃO}

A participação em corridas de rua vem aumentando significativamente nos últimos anos, principalmente motivada por indivíduos que buscam os benefícios para a saúde na pratica regular de exercício físico, numa atividade de fácil execução e baixo custo como a corrida (FREDERICSON; MISRA, 2007; PAZIN et al., 2008; PALUSKA 2005). Tem-se observado um aumento significativo nas lesões em corredores (FREDERICSON; MISRA, 2007), assim como no número de estudos que se preocupam com a prevalência de lesões em corredores.

Porém, esse é o primeiro estudo na literatura em que os corredores são abordados pelos pesquisadores momentos antes de iniciarem sua participação em uma prova. Acreditamos que essa abordagem in loco, nos permitiu avaliar a prevalência de queixa musculoesquelética com uma precisão muito maior do que a forma convencional de aplicação de questionário, que tem o mesmo objetivo, pois acaba entrevistando o corredor em momentos distintos, aumentando a chance de ocorrer um falso/negativo. Esse estudo teve como objetivo analisar a prevalência de dor musculoesquelética em corredores de rua no momento em que precede o início da prova, além de discutir alguns fatores etiológicos relados à prática da corrida.

\section{MATERIAL E MÉTODOS}

\section{PARTICIPANTES}

Os participantes deste estudo foram selecionados aleatoriamente entre os inscritos dos circuitos de corridas de rua, Super $9 \mathrm{~K}$ e Circuito das Estações da cidade de São Paulo no ano de 2009. Foram entrevistados cento e cinquenta e cinco corredores no local das provas, sendo trinta e cinco mulheres $(22,58 \%)$ e cento e vinte homens (77,4l\%). A seleção da amostra se deu por conveniência. Todos os participantes leram e concordaram em assinar um termo de consentimento livre e esclarecido, antes de participar do projeto. O projeto foi aprovado pelo Comitê de Ética da Universidade Cidade de São Paulo.

\section{INSTRUMENTOS}

A entrevista foi composta por um questionário construído pelos próprios autores. O questionário elaborado apresentava questões abertas e fechadas e pode ser dividido em três tópicos principais, sendo eles: os dados pessoais do voluntário (nome, idade, gênero, estatura, peso e contato); o perfil de treinamento, onde constavam questões relacionadas ao tipo de piso predominante no treino (asfalto, grama, esteira, terra e areia), número de treinos por semana e metragem semanal 
de treino e questões relacionadas á presença ou não de dor de origem musculoesquelética naquele momento. No caso de haver uma queixa de dor, perguntava-se sobre a existência de um diagnóstico médico; sobre qual região do corpo a dor estava localizada, sendo que o voluntário poderia apontar a região em um desenho ilustrativo contido no questionário; e ainda, sobre a intensidade desta dor referida, utilizando a Escala Visual Analógica de dor inserida no questionário para mesurar essa intensidade.

\section{PROCEDIMENTO}

O questionário foi preenchido pelos próprios entrevistadores e aplicado momentos antes do início das provas de corrida, em média a entrevista teve a duração de dois minutos. Foi feita uma análise descritiva das variáveis analisadas, além da comparação entre os resultados obtidos entre os grupos através do Teste de Wilcoxon com um $\alpha$ de 0,05 .

\section{RESULTADOS}

Depois da coleta e da análise dos dados, os participantes foram divididos em dois grupos. Um grupo foi formado pelos corredores que apresentavam queixa de dor musculoesquelética no momento da entrevista, e foi intitulado como Grupo com Dor (GD), formado por trinta e nove corredores $(25,16 \%)$. O outro grupo foi composto pelos atletas que não tinham nenhuma queixa, ou dor, composto por cento e dezesseis corredores (74,83\%), sendo intitulado como grupo sem dor (GS).

Observou-se que a média de idade entre os dois grupos foi semelhante, sendo que o GD obteve média de idade de 38,2 anos e o GS de 37,8 anos. Uma vez comparado o IMC, observou-se que o GD obteve a média de IMC de 23,6 e o GS obteve média de 24,3, sendo que a média da estatura foi de 1,74 metros para o GD e I,72 metros para o GS, além de, o peso obter uma média de 7।,8kg para o GD e de $72,6 \mathrm{~kg}$ para o GS.

Em relação às outras variáveis, o tipo de piso predominante durante os treinos para o GD foi à esteira (31 ,48\%), seguida do asfalto $(29,62 \%)$, da grama $(9,25 \%)$, das combinações de piso de asfalto e grama, asfalto e terra e, grama e terra $(5,55 \%)$, seguida ainda, dos pisos de terra, areia e da combinação asfalto e esteira (3,70\%) e, por último o piso com combinação de asfalto e areia ( $1,85 \%)$. Já para o GS o tipo de piso predominante foi o asfalto (57,39\%), seguido da combinação de piso de asfalto e esteira (12,17\%), depois veio a esteira (10,43\%), a combinação com piso de asfalto e terra $(6,95 \%)$ e a terra $(6,08 \%)$ e, por último o piso de areia e a 


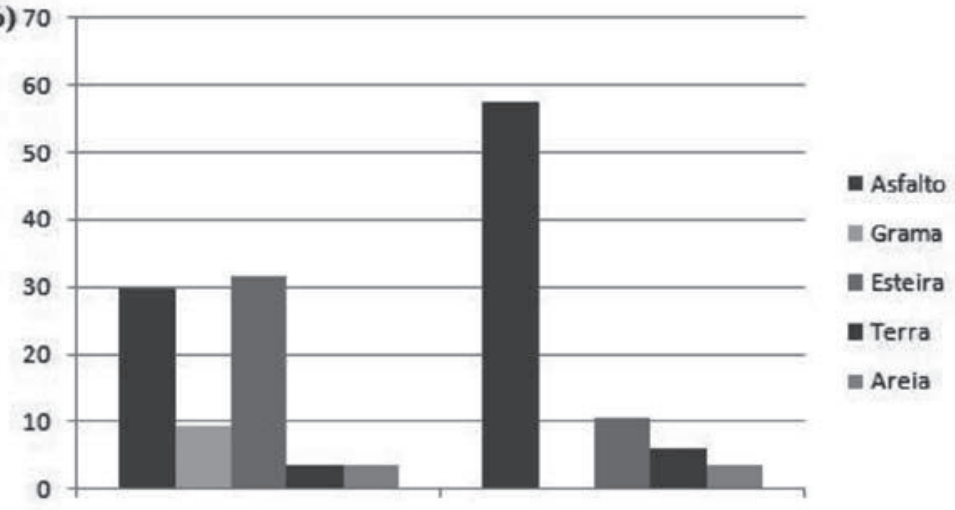

Grupo Dor

GrupoSem Dor

Figura I. Porcentagens de utilização dos diferentes pisos para os dois grupos de corredores.

Sobre a quilometragem semanal foi obtida uma média de 39,6 km para o GD e 30,3 km para o GS, e o número de treinos realizados na semana, teve uma média de quatro treinos/sem. para o GD, e 3,3 treinos/sem. para o GS, como é possível observar na Figura 2. Desta forma, pode-se observar que a prevalência de dor nos corredores participantes teve uma diferença estatisticamente significante, quando comparado com o número de treinos por semana ( $p=0,03)$ e com a quilometragem semanal percorrida $(p=0,0 \mathrm{I})$, porém, não foram observadas diferenças entre as médias de idade e o IMC dos dois grupos.

Figura 2: Diferença entre os dois grupos. M(DP)

\begin{tabular}{lllll}
\hline & Idade & IMC & Km/sem. & Treinos/sem. ${ }^{* *}$ \\
\hline Grupo dor & $38,2(10,2)$ & $23,6(2,6)$ & $39,6(28,2)$ & $4(2,2)$ \\
\hline Grupo sem dor & $37,8(9,9)$ & $24,3(2,5)$ & $30,3(19)$ & $3,3(1)$ \\
\hline
\end{tabular}

* $p=0,03$;** $p=0,01$;

\section{DISCUSSÃO}

Entender os fatores associados à maior prevalência de lesões em corredores é de grande importância para que possíveis medidas preventivas sejam realizadas e para que tenham maior significância. 
Neste estudo a prevalência de lesões musculoesqueléticas momentos antes do inicio da corrida foi de 25, 16\%, valor que se equivale ao encontrado por Van Middelkoop et al. (2008, p. 692) que foi de 28, I\%, onde foram avaliados setecentos e vinte e cinco homens corredores de rua, para descrever a prevalência e a incidência de lesões em MMII, antes e depois da Maratona de Rotterdam e avaliar o impacto destas lesões. Parece que independentemente do tipo de estudo feito, retrospectivo ou prospectivo, com corredores amadores ou não, com corredores de maratona ou de distância menores ( 10 km), no mínimo 20\% - 30\% dos corredores apresentarão algum tipo de queixa ou lesão musculoesquelética.

A média de idade e o IMC, quando comparado entre os grupos com dor e sem dor não obtiveram diferença significativa, estando em coerência com outros estudos (VAN MIDDELKOOP et al., 2008; VAN MIDDELKOOP et al., 2007). A literatura sobre o assunto não é conclusiva sobre essas possíveis relações. Pode-se imaginar que corredores que apresentem um IMC mais elevado possam apresentar uma maior chance de apresentarem lesão musculoesquelética, assim como foi observado por alguns estudos (BUIST et al., 20I0), porém outros estudos mostraram exatamente o contrário (MARTI et al., 1988). Um único estudo relata que indivíduos com mais de $1.70 \mathrm{~m}$ tem um aumento significativo na chance de sofrer lesões, associação essa encontrada num estudo prospectivo com corredores (WEN et al., 1998).

Já em relação ao tipo de piso predominante, quando comparado à prevalência de lesão, os achados deste estudo foram divididos em relação aos grupos GD (esteira - 31,48\%) e GS (57,39\%), sendo assim, em parte, há semelhança destes resultados, com o estudo de Tauton et al. (2003, p. 240), onde foi encontrado o asfalto como piso predominante entre a maioria dos corredores de rua, mas independente da presença ou não de dor. O tipo de superfície onde os indivíduos praticam a corrida não foi considerado como um fator que pode levar ao aumento de lesões. Isso também foi observado na revisão sistemática sobre possíveis fatores que acarretam lesões em corredores (VAN GENT et al., 2007).

Uma das variáveis que apresentou valores estatisticamente significantes relacionados a presença de dor foi à média de quilometragem semanal de treino $(39,6 \mathrm{~km}$ com um $p=0,0 \mathrm{I})$, que se assemelha com os dados encontrados no estudo de Fredericson e Misra (2007, p. 438), onde concluem que acima de aproximadamente $60 \mathrm{~km}$ (40 milhas) de treino por semana, existe um aumento no risco de lesão. A distância semanal percorrida é um dos mais importantes fatores de risco para se desenvolver alguma lesão, conclusão essa que é praticamente um consenso entre os mais diversos estudos sobre lesões em corredores. Na revisão sistemática sobre fatores predisponentes para lesão em corredores, os autores relatam que 
há uma grande evidência entre o surgimento de novas lesões e a distância semanal percorrida. Afirmam também que a evidência de aumento de distância é mais forte para os homens do que para as mulheres, dado esse que vem colaborar com os nossos resultados, pois apenas encontramos possível associação entre a distância percorrida semanal e surgimento de queixas musculoesqueléticas nos homens que responderam o questionário (VAN GENT et al., 2007).

A outra variável analisada que apresentou diferença significativa quando comparada a prevalência de dor nos corredores de rua, foi a média do número de treinos realizados na semana (4 treinos/semana com um $p=0,03$ ), que se aproximam do resultado encontrado em outro estudo (TAUNTON et al., 2003), onde a frequência de treinamento teve a maior porcentagem nos treinos realizados três vezes por semana (59,6\%). E ainda, neste mesmo estudo de Taunton et al. (2003, p. 24I) foi visto que para as mulheres, treinar apenas uma vez por semana, aumenta o risco de lesão neste público.

Ainda, embora as outras variáveis analisadas neste estudo não tenham demonstrado estatisticamente uma relevância significativa, elas não devem ser descartadas em estudos futuros, por estarem diretamente envolvidas, quando observadas na prática clínica. Entendemos que são necessários mais estudos nesta área designados á este público, mas acreditamos que esse estudo tenha servido de grande importância para evidenciar os fatores associados á prevalência de dor nesta população, o que nos deixa bastante atento, uma vez que, mais de um quarto da população de corredores, participa das provas de corrida, referindo algum tipo de dor.

Os resultados deste estudo devem ser observados com cautela em função das limitações que o nosso trabalho apresenta. A principal limitação do nosso estudo é que a forma para se mensurar a prevalência de corredores com queixas ou dores de origem musculoesqueléticas foi através do uso de um questionário, não havendo nenhum tipo de avaliação clínica pelos avaliadores. Sabemos que a pergunta pode ser interpretada de diferentes maneiras pelos corredores, sem contar o fato que a dor é um aspecto subjetivo de difícil mensuração.

\section{CONCLUSÃO}

A prevalência de dor em corredores de rua no momento em que precede o início da prova, que foi de aproximadamente $25 \%$ nesse estudo, está diretamente associada ao volume de treino semanal, ou seja, tem relevância significativa, quando relacionada à quilometragem semanal de treino e á quantidade de treinos realizados por semana. 


\section{Prevalence of pain in runners at the time in which precede the beginning of the race}

ABSTRACT: The participation in running has increase significantly motivated by the health benefits, easy execution and low cost. The aims of this study were to calculate the prevalence of presence of musculoskeletal pain in recreational runners immediately before a race and discuss the some hypothesis of it. The participants were divided into two groups. The first one composed for runners with musculoskeletal pain complaints and the other group without any complaints. We found a statistical relationship when compare the number of training per week $(p=0,03)$ and weekly distance $(p=0,01)$. We conclude that prevalence of pain was about $25 \%$ and was directly associated with weekly training volume.

KEYWORDS: Running; pain; prevalence; sports.

\section{Prevalencia del dolor en los corredores en el momento anterior al inicio de la carrera}

RESUMEN: La participación en las carreras de atletismo de larga distancia que se realizan en circuitos urbanos ha aumentado considerablemente en los últimos años, motivada por los beneficios en la salud, su fácil ejecución y su bajo costo. Este estudio tuvo como objetivo analizar la prevalencia de dolor musculoesquelético en corredores previo al inicio de las carreras, y discutir algunos de los factores etiológicos relacionados con la práctica de las carreras de larga distancia. Los participantes se dividieron en dos grupos; un grupo estaba compuesto por los corredores que indicaron presencia de dolor musculoesquelético y el otro grupo por los que no presentaron dolor. Se encontró una diferencia estadísticamente significativa al comparar el número de entrenamientos por semana $(p=0,03)$ y el kilometraje recorrido semanalmente $(p=0,0$ I). Se concluye que la prevalencia de dolor fue alrededor del $25 \%$, asociada directamente con el volumen de entrenamiento semanal.

PALABRAS CLAVE: Carrera; dolor; prevalencia; deportes.

\section{REFERÊNCIAS}

BUIST, I. et al. Incidence and risk factors of running-related injuries during preparation for a 4-mile recreational running event. British Journal of Sports Medicine, Groningen, v. 44, n. 8 , p. 598-604, 2010.

FREDERICSON, M.; A. K. Misra. Epidemiology and aetiology of marathon running injuries. Sports Medicine, v. 37, n. 4-5, p. 437-439, 2007. 
MARTI, B. et al. On the epidemiology of running injuries. The 1984 Bern Grand-Prix study. American Journal of Sports Medicine, v. 16, n. 3, p. 285-294, 1988.

PALUSKA, S. A. An overview of hip injuries in running. Sports Medicine, v. 35, n. II, p. 99|-1014, 2005.

PAZIN, J. et al. Corredores de rua: características demográficas, treinamento e prevalência de lesões. Revista Brasileira de Cineantropometria e Desempenho Humano, v. 10, n. 3, p. 277-282, 2008.

TAUNTON, J. E. et al. A prospective study of running injuries: the Vancouver Sun Run "In Training" clinics. British Journal of Sports Medicine, v. 37, n. 3, p. 239-244, 2003.

VAN GENT, R. N. et al. Incidence and determinants of lower extremity running injuries in long distance runners: a systematic review. British Journal of Sports Medicine, v. 4I, n. 8, p. 469-480; discussion 480, 2007.

VAN MIDDELKOOP, M. et al. Prevalence and incidence of lower extremity injuries in male marathon runners. Scandinavian Journal of Medicine; Science in Sports, v. 18, n. 2, p. | 40-144, 2008.

VAN MIDDELKOOP, M. et al. Course and predicting factors of lower-extremity injuries after running a marathon. Clinical Journal of Sport Medicine, v. I7, n. I, p. 25-30, 2007.

WEN, D. Y. et al. Injuries in runners: a prospective study of alignment. Clinical Journal of Sport Medicine, v. 8, n. 3, p. 187-194, 1998.

Recebido: 14 mar. 2010 Aprovado: 08 dez. 2010

Endereço para correspondência: Universidade Cidade de São Paulo (UNICID) Rua Cesário Galeno, 448/475 Bairro Tatuapé CEP: 0307। -000 São Paulo - SP 